\title{
OPTICAL OBSERVING TECHNIQUES FOR FAINT EXTENDED SURFACE BRIGHTNESS
}

\author{
Gerhard F. O. Schnur \\ Astronomical Institute \\ Ruhr-University Bochum \\ P.O. Box 102148 \\ D-4630 Bochum 1 \\ FRG
}

\author{
Kalevi Mattila \\ Helsinki University Observatory \\ Tähtitorninmäki \\ SF-00130 Helsinki \\ Finland
}

\begin{abstract}
The measurement of faint extended surface brightness at a level of a few $S_{10}$ is hampered by the disturbingly bright contributions of the Light of the Night Sky (LONS), consisting of, among others, the zodiacal light, airglow, and integrated starlight. Special observing techniques are necessary for investigations of the diffuse light in clusters of galaxies (DLCG) and for the determination of the extragalactic background light (EBL). We argue for the two-telescope method as an effective means to compensate for the bright components of the LONS, which will allow an accurate determination of faint extended surface brightnesses.
\end{abstract}

\section{INTRODUCTION}

The lowest values for the individual components of the LONS have been reported by Roach and Gordon (1973), and by Mattila (1989), under nearly ideal circumstances for observations of faint extended surface brightness, i.e., integrated starlight (ISL) at the galactic pole, zodiacal light (ZL) at the ecliptic pole, airglow (AG) at zenith position, and only a minor contribution of atmospheric scattered light (ASL) at the zenith. Despite these fortunate conditions the sum of these components (ISL + ZL + AG + ASL) amounts to 150-170 units $\left(1 \times 10^{-9}\right.$ erg $\mathrm{cm}^{-2} \mathrm{~s}^{-1} \mathrm{sr}^{-1} \AA^{-1}$ ), while the expected contributions from the components of the diffuse galactic light (DGL) are $\leq 10$ and those of the EBL are very likely even less. To determine the EBL and/or the DGL, the other bright components have to be known to better than $1 \%$ for accurate subtraction.

\section{THE TWO-TELESCOPE OBSERVING TECHNIQUE}

The main disturbing component of the LONS for ground based observations is the airglow, with its patchiness and variability over time. If only one telescope is used for On and Off source measurements, in order to observe differentially, the variation of the AG during the switch from the On to the Off position prevents the attainment of the necessary accuracy. Continuous monitoring of the AG variations is needed while doing On/Off measurements.

For our observations of the EBL and for our search for diffuse light in clusters of galaxies, we have developed the Two Telescope Observing Technique. Two telescopes are used, equipped with nearly identical photometers. Two identical sets of filters are installed in the two photometers. The individual integrations are synchronized in time to an accuracy of about $1 \mathrm{~s}$ over a typical measuring cycle of $300 \mathrm{~s}$. 
The reference position for the On/Off measurements is selected so that it is free of stars down to the plate limit of the ESO/SRC Schmidt Atlas. Furthermore this position on the sky is selected so that even in the region surrounding the reference position there are as few stars as possible. The photometer of the smaller telescope is equipped with the largest possible aperture, allowing it to obtain a very accurate determination of the airglow through each of the different filters. The effective field of the smaller telescope was about $6^{\prime}$ in diameter, while the large telescope had a field $88^{\prime \prime}$ in diameter. The simultaneous measurements at the reference point with both telescopes permit an accurate determination of the ratio of sensitivity between the two detectors. The signal varied by more than $50 \%$ during a few hours observing. The ratio, however, remained accurate to better than $1 \%$, permitting close cancellation of the airglow component for successive On/Off measurements. The accuracy of our observations is determined only by the photon noise of the measurements within the 88 " diaphragm at the large telescope. The remaining small background gradients for ZL and AG can be subtracted by fitting a $\csc b$ model through the background areas which extend over $3-4^{\circ}$. A detailed description of our observing technique is given by Mattila and Schnur (1988).

\section{PRELIMINARY RESULTS ON THE EBL AND DLCG}

First results for the observed EBL are reported by Mattila (1989) at the position of the L1642 high-galactic-latitude cloud: $\mathrm{EBL}=6.5 \pm 2.5 \times 10^{-9} \mathrm{erg} \mathrm{cm}^{2} \mathrm{~s}^{-1} \mathrm{sr}^{-1} \AA^{-1}$. For the DLCG we report preliminary results for the Abell cluster A1146 in Table 1. A comparison of the colors of the DLCG with those of the central CD galaxy shows that the DLCG is rather blue. Final data on our EBL and DLCG are presented by Mattila and Schnur (1989, this volume).

TABLE 1. DLCG in A1146 observed in the uvgr-system. The numbers are averages of six positions, each observed 6-9 times. The signal from the central $\mathrm{cD}$ galaxy as seen in the $88^{\prime \prime}$ diaphragm is given for comparison.

\begin{tabular}{lccccc}
\hline \hline & $u$ & $v$ & $g$ & $r$ & unit \\
\hline DLCG & $1.7 \pm 1.2$ & $2.2 \pm 0.3$ & $1.7 \pm 0.6$ & $3.2 \pm 0.6$ & $S_{10}$ \\
cD galaxy & - & $9.3 \pm 4.2$ & $20.1 \pm 1.9$ & $44.4 \pm 3.0$ & $S_{10}$ \\
\hline
\end{tabular}

\section{REFERENCES}

Mattila, K. 1989, Proc. IAU Symp. No. 139, Galactic and Extragalactic Background Radiation, ed. S. Bowyer and Ch. Leinert (Dordrecht: Kluwer Academic Publisher).

Mattila, K. Schnur, G. F. O. 1988, ESO Messenger, 51, 34.

Roach, F. E. and Gordon, J. L. 1973, The Light of the Night Sky, D. Reidel Publ. Comp.: Dordrecht/Boston. 